\title{
Economical point plotter interface for a PDP-12 computer
}

\author{
DENNIS E. UPRIGHT and RAYMOND E. BANNAR \\ Maryland Psychiatric Center, Box 3235, Baltimore, Md. 21228
}

An economical point plotter interface is described for the PDP-12 computer.

Efficient high-speed analyses of data processed by means of a small laboratory computer such as a PDP-12 frequently requires automatic point plotting. Unfortunately, the cost of a commercial plotter with requisite controller and digital-to-analog (D/A) converter may be a substantial portion of the cost of the system. Several point plotters below $\$ 1,000$ are commercially available and need only to be interfaced with the PDP-12 to provide an efficient low-cost system. This note describes the design and construction of the interface required between the PDP-12 and a Hewlett-Packard 7004B X-Y recorder.

The recorder was purchased with two Model 17178A dc attenuators (one for each axis), a Model 17173A null detector, and a remote-controlled pen (Model 17012B). The total cost of the plotter with these accessories is $\$ 1,965$. The operation of this assembly is as follows: Upon receipt of a "seek" pulse, the null detector enables the plotter servos and moves to the point represented by the two analog voltages presented to the $X-Y$ inputs. At the representative point, it drops the remote-control ink pen onto the paper, turns off the servo control motors, and issues a "plot complete" pulse. The same device will accept an "enable-disable" pulse, permitting the entire plotter to be put into "on" or "off" line operation by means of the computer program.

The commercially available plotter control card offered by DEC for the PDP-12 (M704), which occupies Card Location MN05, and the cable card, which occupies Location M04, will not drive the HP X-Y plotter. These cards were designed to interface other makes of incremental plotters. However, all required signals for the instruction codes are present at these connectors.

Since the PDP-12 contains digital-to-analog converters (DACs) to drive the VR-12 display oscilloscope, these may be used to drive the X-Y plotter. The intensity pulse issued by the DIS instruction can serve as the seek pulse after buffering. Three instructions are required: (1) enable device, (2) disable device, and (3) skip if device is ready.

Figure 1 shows the schematic of the required interface. The BMB lines are decoded as shown to produce a device-select code of 650 . This 650 code is

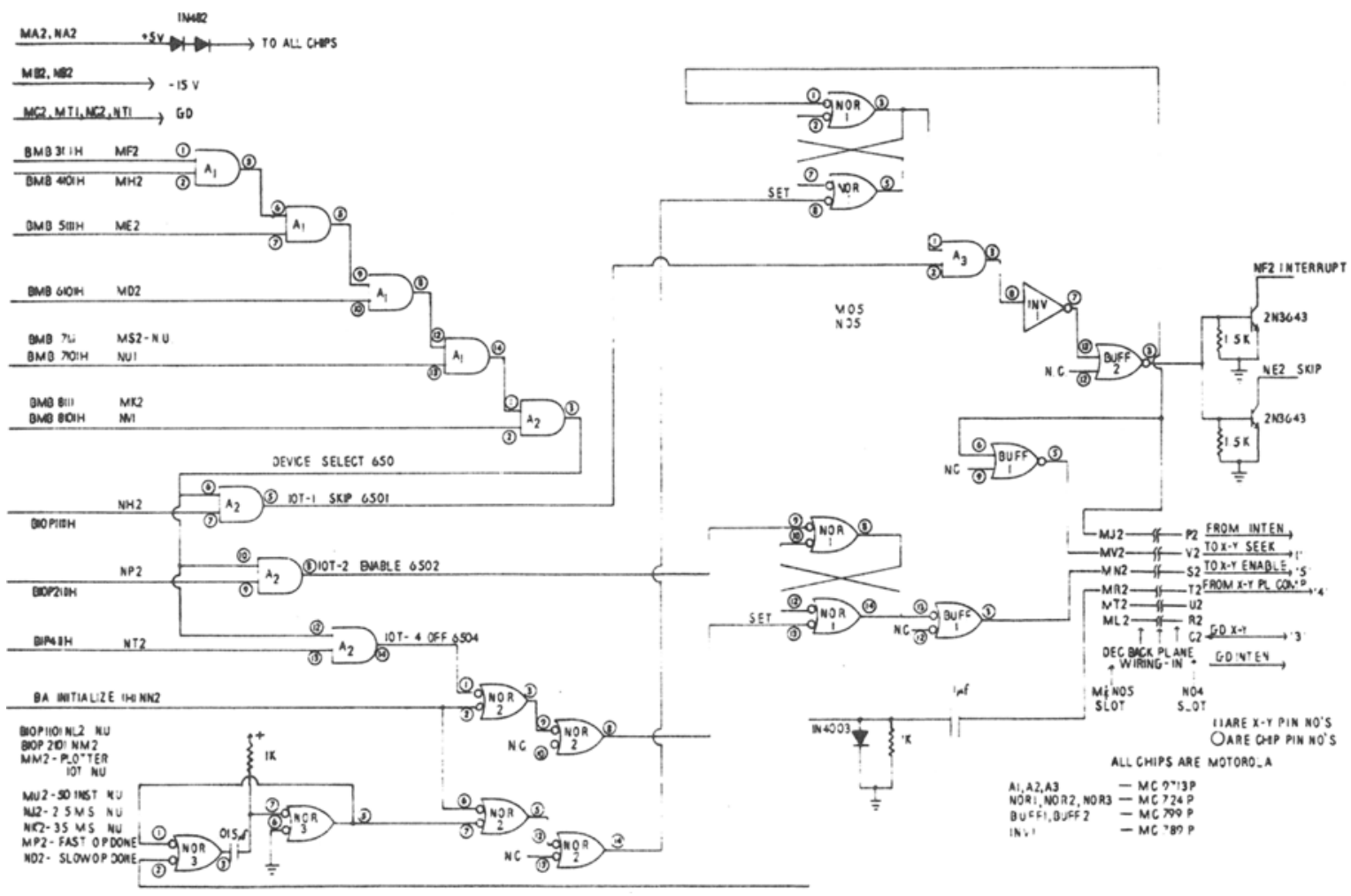

Fig. 1. 


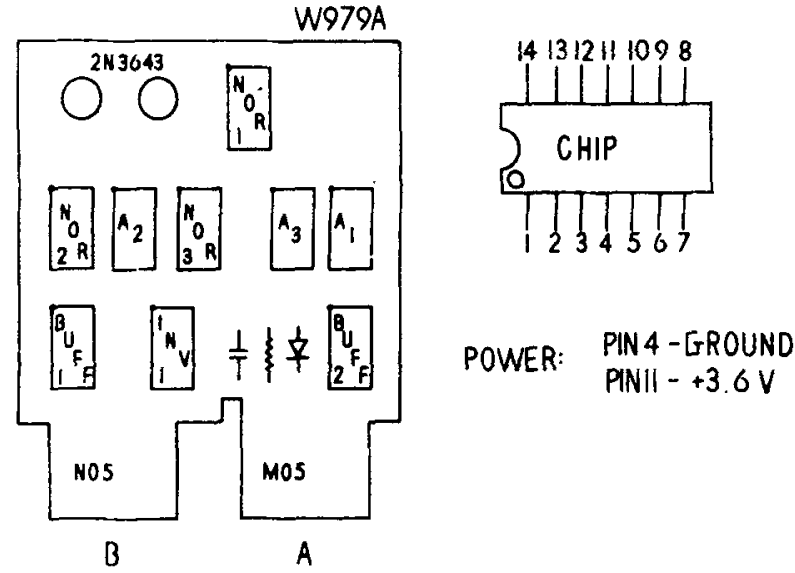

Fis. 2.

then ANDed with the BIOP lines to create the three required instructions, 6501 (on IOT-1) for the skip, 6502 (on IOT-2) for the enable, and 6504 (on IOT-4) for the disable.

The remaining logic functions as follows: The BA initialize (IO preset) pulse is NORed with the 6504 instruction to set the NOR 1 flip-flop, causing Pin 14 to go low and the output of the buffer to go high, which disables the plotter. Similarly, the 6502 instruction will reset the flip-flop, thus reversing the sequence and enabling the plotter.

The 6504 instruction is also NORed with the plot complete pulse (through a one-shot) and it is used to set the upper NOR 1 flip-flop. The flip-flop output on Pin 3 will be high, thus allowing A 3 to pass the 6501 skip instruction to the skip and interrupt bus drivers (i.e., the device is ready).

An intensity pulse from the DIS instruction will issue an X-Y seek pulse through BUFF 1 and will reset the flip-flop, thus inhibiting a skip or interrupt. The plot complete pulse is passed through the NOR 3 one-shot to sharpen it and to insure its duration.

Since DEC wiring is installed between NO 4 and M and N0 5, only a blank cable card need be wired and installed in $\mathrm{N} 4$ to control the plotter. Of course, the DAC outputs and intensity pulse must be wired from the auxiliary scope connector.

Figure 2 shows the top view of the W979A card with the chip and discrete component placement.

Approximate cost of additional components (excluding plotter) is $\$ 40$.

By installing the necessary logic on a DEC W979A blank card and the interface cabling on a DEC M 904B memory-buss connector card, these card slots can be utilized. Therefore, it is not necessary to use slots $\mathrm{N} 13$, $\mathrm{N} \mathrm{14}$, and $\mathrm{N} 15$ (IOT connectors) or to daisy-chain the slots to other locations.

(Received for publication October 3, 1972; accepted October 27, 1972.) 